\title{
Distribución geográfica de la enfermedad de Kawasaki en Chile
}

\author{
Rodrigo Hoyos-Bachiloglu, Álvaro García, Pamela S. Morales, \\ Jaime Cerda, Eduardo Talesnik y Arturo Borzutzky
}

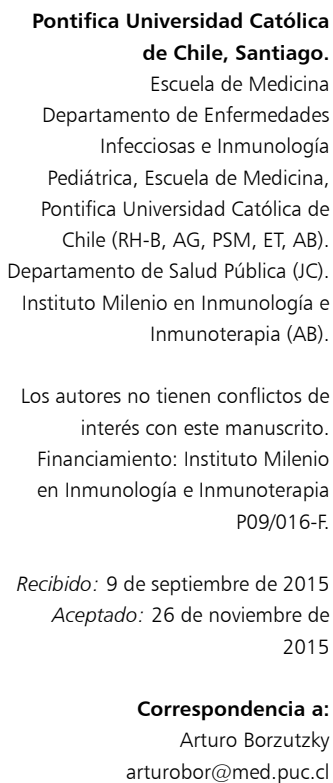

Pontifica Universidad Católica de Chile, Santiago. Escuela de Medicina Departamento de Enfermedades Infecciosas e Inmunología Pediátrica, Escuela de Medicina, Pontifica Universidad Católica de

Chile (RH-B, AG, PSM, ET, AB) Departamento de Salud Pública (JC) Instituto Milenio en Inmunología e Inmunoterapia (AB).

Los autores no tienen conflictos de interés con este manuscrito. Financiamiento: Instituto Milenio en Inmunología e Inmunoterapia P09/016-F.

Recibido: 9 de septiembre de 2015 Aceptado: 26 de noviembre de

Correspondencia a: Arturo Borzutzky arturobor@med.puc.c

\begin{abstract}
Geographic distribution of Kawasaki disease throughout Chile
Background: Incidence of Kawasaki disease (KD) in Chile is rising, however the distribution of cases throughout Chile is unknown. Objective: To describe the epidemiology of KD in Chile between years 2001 and 2011, and study the geographic distribution of KD cases throughout the country. Methods: We reviewed national hospital discharge databases for KD cases (ICD10 code M30.3) in children $<18$ years. KD admission rates per 100,000 children $<5$ years were calculated for every commune, health district and region, as a proxy of KD incidence. Results: $1,404 \mathrm{KD}$ cases were registered with a national KD incidence rate of 8.7. KD incidence rate increased significantly from 5.9 in 2001-2003 to 10.4 in 2009-2011 ( $p<0.001$ ). Regions IX (Araucanía), Metropolitan and VI (O'Higgins) had the highest KD incidence (12.4, 11.1 and 10.5 respectively), and regions III (Atacama), II (Antofagasta) and XII (Magallanes), had the lowest incidence (0.8, 3.9 and 4, respectively). The Eastern Metropolitan Health District, the population with the highest socioeconomic status in Chile, had the highest KD incidence rate (19.8) and concentrated $23.9 \%$ of the country's hospital discharges for KD. Conclusion: KD incidence in Chile is heterogeneous, with concentration of caseloads in the central regions and especially in the Eastern Metropolitan Health District. Geographic variations of KD in Chile could be associated with real differences in incidence or with disparities in diagnostic opportunity, and access to specialists and tertiary healthcare centers.

Key words: Kawasaki disease, epidemiology, socioeconomic status, Chile.

Palabras clave: Enfermedad de Kawasaki, epidemiología, Chile, nivel socio-económico.
\end{abstract}

\section{Introducción}

L a enfermedad de Kawasaki (EK) es una enfermedad febril autolimitada descrita inicialmente por el Dr. Tomisaku Kawasaki en $1967^{1}$. Además de la fiebre de duración mayor a 5 días, se caracteriza por síntomas y signos clínicos clásicos como exantema, conjuntivitis, cambios en mucosas, cambios en extremidades, adenopatía cervical y parámetros inflamatorios elevados. Histopatológicamente constituye una vasculitis de vaso mediano con predilección por las arterias coronarias, siendo hoy en día la causa más frecuente de cardiopatía adquirida en la población pediátrica en países desarrolla$\operatorname{dos}^{2}$. Estudios poblacionales han descrito la epidemiología de la EK en distintos países, principalmente del este de Asia, Europa y América del Norte ${ }^{3}$. La mayoría de estos estudios muestran que la EK se presenta predominantemente en niños bajo 5 años de edad y que existe una mayor proporción de afectados de sexo masculino 3 .

La etiología de la EK aún permanece desconocida, siendo una de las teorías aceptadas actualmente la de una infección por agente desconocido en hospederos genéticamente susceptibles. Esta teoría del origen infeccioso de la EK se basa en datos epidemiológicos obtenidos por medio de los egresos hospitalarios por EK, encuestas nacionales o reportes de esta enfermedad, y datos clínicos, que asemejan el cuadro clínico de la enfermedad con los síntomas y el comportamiento de infecciones virales y bacterianas. Existen síntomas similares entre EK e infección por adenovirus y escarlatina, variaciones estacionales de incidencia ${ }^{4}$, brotes epidémicos ${ }^{5-7} y$ agrupación espacio-temporal de los casos ${ }^{8}$.

La incidencia de la EK ha ido aumentando de forma sostenida en muchos países del mundo ${ }^{7,-11}$. La incidencia reportada varía dependiendo de factores geográficos, étnicos y socio-económicos. El efecto del nivel socioeconómico sobre la incidencia de EK ha sido reportada principalmente durante períodos de brotes epidémicos de $\mathrm{EK}^{5,6}$, aunque su efecto sobre la incidencia de EK no es clara en países como Japón ${ }^{12}$. Un estudio descriptivo de la epidemiología de la EK en Chile entre los años 2001 y 2007 mostró que la tasa de incidencia de EK en Chile se ha incrementado gradualmente, estimándose una tasa de incidencia de 8,4 casos por 100.000 niños bajo 5 años entre 2005 y $2007^{13}$. De acuerdo con este mismo estudio, la Región Metropolitana (RM) concentró cerca de la mitad de los casos de EK en el período estudiado y la población adscrita al sistema de salud privado concentraría una 
mayor proporción relativa de casos de EK en nuestro país comparada con la población beneficiaria del sistema público (FONASA). Sin embargo, no se ha realizado un análisis detallado de la distribución geográfica de la EK en Chile. El objetivo de este trabajo es describir la epidemiología de la EK en Chile entre 2001 y 2011 y estudiar la distribución geográfica de los casos de EK a lo largo del país.

\section{Métodos}

Se realizó una revisión de las bases de datos de egresos hospitalarios del Departamento de Estadísticas e Información de Salud del Ministerio de Salud de Chile entre los años 2001 y 2011. Al momento del análisis de datos del presente estudio, no se encontraban disponibles las bases de datos de años posteriores al 2011. Se consideró para el estudio aquellos casos codificados como EK (código ICD-10 M30.3) en pacientes bajo 18 años de edad. La unidad de análisis de la base de datos utilizada es hospitalizaciones y no pacientes, dado que ésta no contiene identificadores de pacientes únicos. Por lo tanto, no considera re-hospitalizaciones por EK o casos de EK que nunca fueron hospitalizados. El análisis de los egresos hospitalarios por EK ha sido utilizado previamente tanto a nivel nacional como internacional para estimar la incidencia de la enfermedad.

Se calculó la tasa de hospitalizaciones por EK por 100.000 habs. bajo 5 años de edad para cada región, Servicio de Salud (SS) y comuna del país. Considerando la creación de dos nuevas regiones (XIV-Arica/Parinacota y XV-De los Ríos) en nuestro país en el año 2007, los análisis regionales fueron realizados considerando el sistema de 15 regiones para todo el período con tal de mantener las variables geográficas del estudio constante. Los datos de egresos hospitalarios correspondientes a la RM fueron además analizados según SS de egreso hospitalario de los pacientes, es decir el SS donde fue dado de alta cada paciente hospitalizado por EK, de manera independiente de su lugar de residencia.

El presente estudio fue aprobado por el Comité de Ética de Investigación de la Escuela de Medicina de la Pontificia Universidad Católica de Chile.

\section{Análisis estadístico}

Todas las tasas se muestran con intervalo de confianza (IC) del 95\%. Los cambios temporales en incidencia de EK fueron analizados mediante regresión lineal simple. Para evaluar el exceso o déficit de casos de EK por región, se calculó la razón de incidencia estandarizada (RIE) de EK para cada región de Chile. Este razón permite comparar el número de casos observados contra los casos esperados utilizando la tasa de incidencia nacional como referencia $(\mathrm{RIE}=1,0)$. Un valor $\mathrm{p}<0,05$ fue considerado estadísticamente significativo. Los análisis estadísticos fueron realizados con los software SPSS versión 21.0 (IBM Corporation, Armonk, NY) y OpenEpi versión 2.3.1 (www.OpenEpi.com, actualizado el 23/06/2011).

\section{Resultados}

Entre los años 2001 y 2011 se registraron en Chile 1.404 hospitalizaciones con diagnóstico de egreso correspondiente a EK. Las características demográficas de estos egresos se muestran en la Tabla 1. La edad mediana al diagnóstico fue de 1 año; 1.204 casos correspondieron a pacientes bajo 5 años de edad, representando $85,7 \%$ de los casos de EK. La relación hombre: mujer fue de 1,7:1. La tasa de egresos hospitalarios por EK estimada para el período fue de 8,7 casos por 100.000 habs. bajo 5 años (IC 95\% 8,2-9,2). Se observó un aumento significativo de incidencia de EK desde el trienio 2001-2003 al trienio 2009-2011 desde 5,9 a 10,4 por 100.000 habs. bajo 5 años $(\mathrm{p}<0,001)$. Sólo dos muertes con diagnóstico de enfermedad de Kawasaki al egreso se produjeron en el período, resultando en una letalidad hospitalaria de $0,1 \%$.

$\mathrm{Al}$ analizar la distribución regional de EK en Chile, se observó una alta concentración de casos en la zona centro del país, destacando que casi $50 \%$ de los casos nacionales de EK se registraron en la RM (n: 691). Las tasas de incidencia de EK para cada región del país se muestran en la Figura 1. Las mayores tasas de incidencia de EK a nivel nacional se registraron en las regiones de La Araucanía (IX), Metropolitana (RM) y O'Higgins (VI) $(12,4 ; 11,1$ y 10,5 por 100.000 habs. bajo 5 años, respectivamente). Las regiones con las menores tasas de incidencia de EK registradas fueron las regiones Atacama (III), Antofagasta (II) y Magallanes (XII) $(0,8 ; 3,9$ y 4 por 100.000 habs. bajo 5 años, respectivamente).

Tabla 1. Características demográficas de los egresos por enfermedad de Kawasaki en Chile entre los años 2001 y 2011 (n: 1.404)

\begin{tabular}{ll}
\hline Hombres, n (\%) & $883(62,9)$ \\
Mujeres, n (\%) & $521(37,1)$ \\
\hline Edad & \\
\hline Mediana (rango), años & $1(0-17)$ \\
$<1$ año, n (\%) & $354(25,2)$ \\
1 a 4 años, n (\%) & $850(60,5)$ \\
5 a 9 años, n (\%) & $154(11)$ \\
10 a 17 años, n (\%) & $46(3,3)$ \\
Previsión & $890(63,4)$ \\
\hline FONASA, n (\%) & $514(36,6)$ \\
ISAPRE/Particular, n (\%) & $2(0,1)$ \\
\hline
\end{tabular}


El análisis de la RIE por región determinó que sólo las regiones de La Araucanía (RIE 1,43; IC 95\% 1,091,76) y RM (RIE 1,27; IC 95\% 1,16-1,38) presentan una incidencia de EK significativamente por sobre la tasa nacional de EK, mostrando también una tendencia a

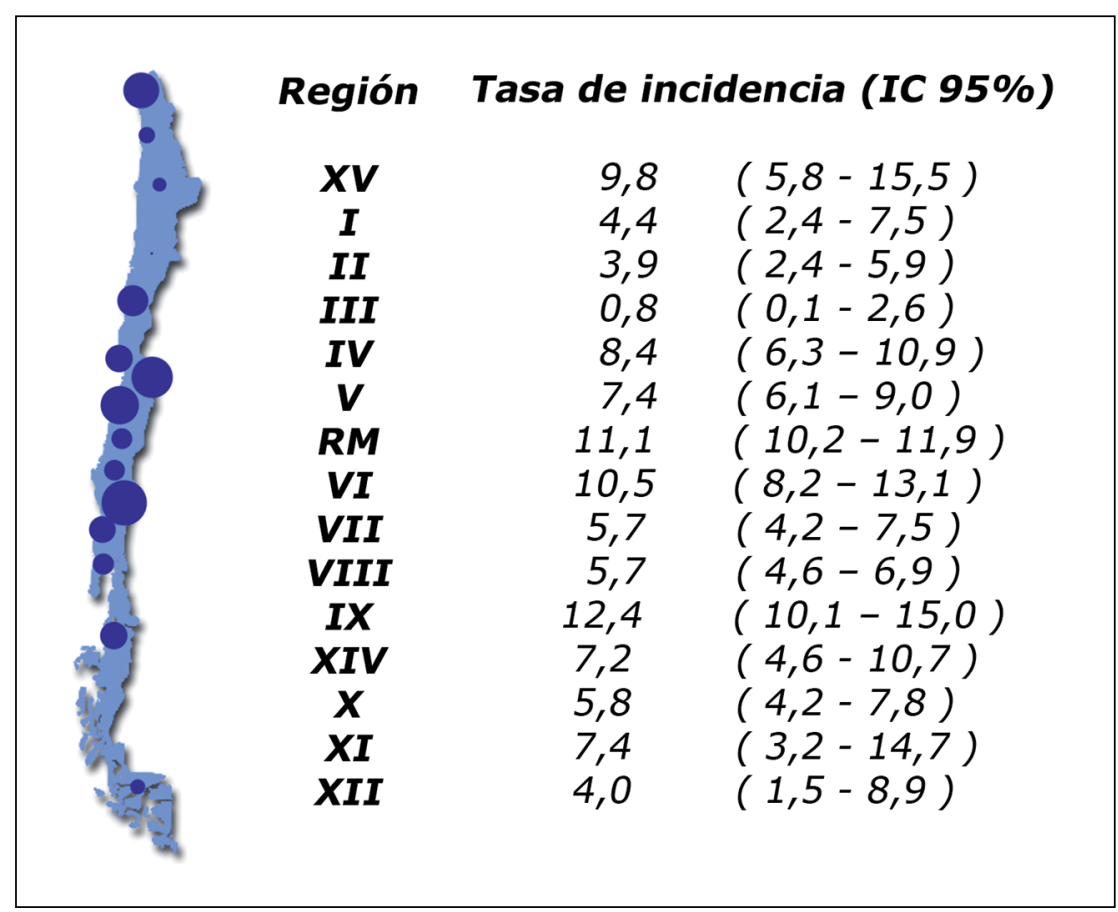

Figura 1. Tasas de incidencia regionales de EK por 100.000 habs. bajo 5 años de edad, 2001-2011. El tamaño de los círculos en el mapa representa la incidencia regional. mayor diagnóstico en la zona centro del país comparado con las zonas norte y sur (Figura 2). Se observó RIE bajo el promedio nacional en las regiones Arica/Parinacota (I), Antofagasta, Atacama, Maule (VII), Bío-Bío (VIII), Los Lagos (X) y Magallanes.

Los egresos por EK fueron luego analizados por SS, tanto de residencia del paciente como de egreso hospitalario (SS donde está localizado geográficamente el centro hospitalario). Los SS con mayor tasa de incidencia de EK en el país fueron el SS Metropolitano Oriente (SSMO) con 19,8 y el SS Araucanía Sur con 13,9 casos/100.000 niños bajo 5 años de edad. A su vez los SS con menor tasa de incidencia de EK fueron los de Atacama $(0,8)$ y Bío-Bío $(2,1)$. En la RM, se registraron 691 egresos por EK según SS de residencia y 787 según SS de egreso. De entre los pacientes con residencia en la RM, dos fueron dados de alta en otras regiones, mientras que 98 de 713 casos de EK con residencia fuera de la RM (13,7\%) egresaron de SS localizados en esta última. En la RM, el SSMO concentró el mayor porcentaje de egresos hospitalarios por EK por SS de residencia (14\% del total del país), seguido por los SS Metropolitano Suroriente $(11,1 \%)$ y Metropolitano Central (8,1\%) (Figura 3A). En tanto, según SS de egreso hospitalario, el SSMO concentró $23,9 \%$ de los casos nacionales seguido por el SS Metropolitano Central con $10,1 \%$, reflejando migración de otros SS hacia estos para diagnóstico y/o tratamiento hospitalario (Figura 3B).

En cuanto a distribución por comunas de Chile y en concordancia con datos anteriormente mostrados, $60 \%$ de las comunas con mayor número de casos se encuentran en la RM. Las comunas con mayor número de casos del país
Figura 2. Razón de incidencia estandarizada regional de EK, 2001-2011.

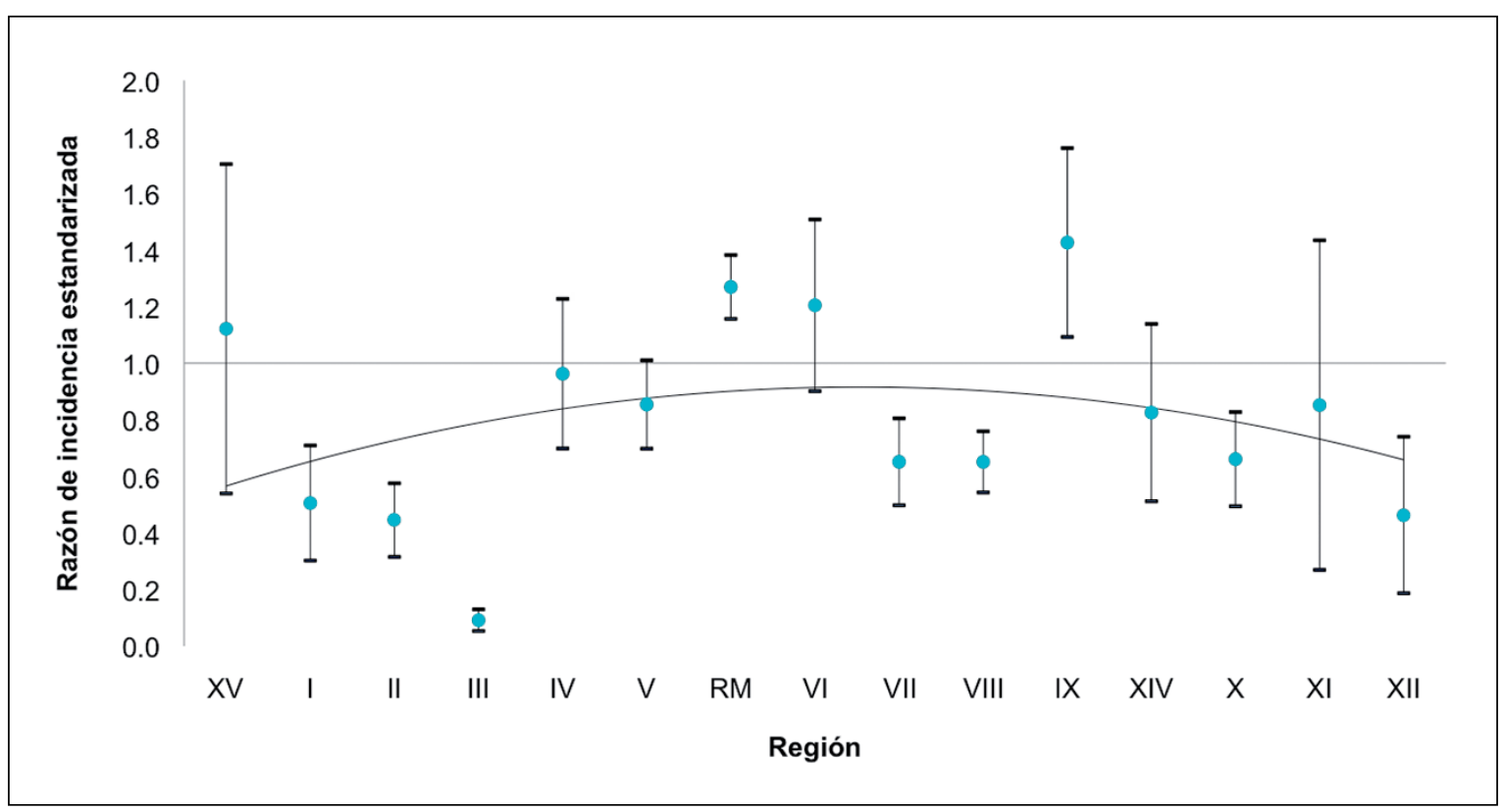




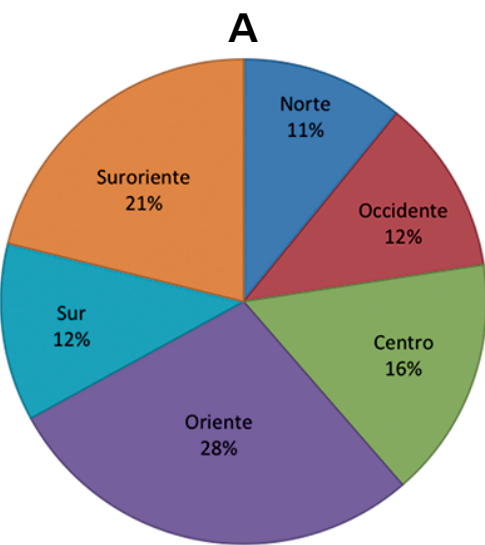

B

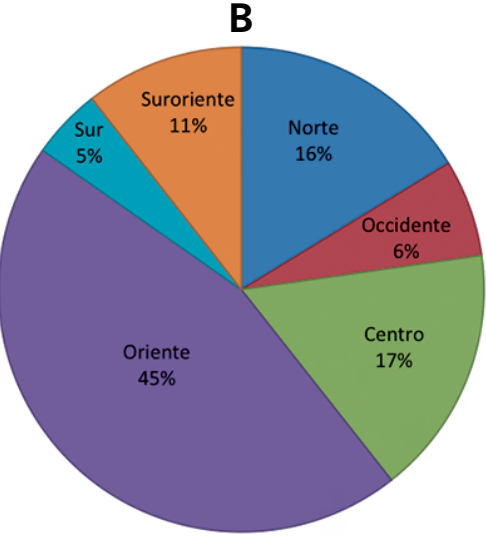

Figura 3. Distribución de los egresos hospitalarios por EK en la Región Metropolitana, 2001-2011. A) Distribución de casos de EK según servicio de salud de residencia (n: 691). B) Distribución de casos de EK según servicio de salud de egreso hospitalario (n: 787).

fueron Puente Alto, Maipú y La Florida, las tres comunas más pobladas del país (Tabla 2). En cambio, las comunas de mayor incidencia del país fueron Cunco y Traiguén con tasas muy por sobre la tasa global nacional (Tabla 3). No obstante lo anterior, dos tercios de las comunas de mayor incidencia del país de todas formas se encontraron en la $\mathrm{RM}$, incluyendo todas las comunas del SSMO.

\section{Discusión}

El presente estudio muestra la epidemiología de la EK en Chile entre 2001 y 2011 y la distribución de los egresos hospitalarios por EK a lo largo del país, encontrando una marcada asimetría entre los sectores norte, centro y sur. El sector centro, principalmente la RM y el SSMO, concentró la mayor proporción de egresos por EK en el período estudiado. Posibles explicaciones para estas variaciones en
Tabla 2. Comunas de Chile con mayor número de casos de EK reportados en Chile, 2001-2011

\begin{tabular}{|lcc|}
\hline Comuna & Casos $(\mathbf{n})$ & \% del total país \\
\hline Puente Alto & 74 & 5,3 \\
\hline Maipú & 60 & 4,3 \\
\hline La Florida & 44 & 3,1 \\
\hline Peñalolén & 43 & 3,1 \\
\hline Rancagua & 38 & 2,7 \\
\hline Las Condes & 38 & 2,7 \\
\hline Temuco & 37 & 2,6 \\
\hline Santiago & 36 & 2,6 \\
\hline Nuñoa & 34 & 2,4 \\
\hline La Serena & 31 & 2,2 \\
\hline San Bernardo & 28 & 2,0 \\
\hline Chillán & 28 & 2,0 \\
\hline Viña del Mar & 26 & 1,9 \\
\hline Providencia & 20 & 1,4 \\
\hline Talca & 20 & 1,4 \\
\hline Quilicura & 20 & 1,4 \\
\hline
\end{tabular}

Tabla 3. Comunas de Chile con las mayores tasas de incidencia de EK en $<5$ años reportadas en Chile, 2001-2011

\begin{tabular}{|lccc}
\hline Comuna & Casos $\mathbf{2} \mathbf{5}$ años $\mathbf{( n )}$ & $\begin{array}{c}\text { Tasas de Incidencia EK } \\
\text { por } \mathbf{1 0 0 . 0 0 0} \text { niños bajo } \\
\mathbf{5} \text { años }\end{array}$ & IC 95\% \\
\hline Cunco & 16 & 102,9 & $60,9-163,6$ \\
\hline Traiguén & 6 & 41,7 & $16,9-86,7$ \\
\hline Santiago & 34 & 29,9 & $21,1-41,3$ \\
\hline Nuñoa & 25 & 27,6 & $18,2-40,1$ \\
\hline Vitacura & 15 & 26,7 & $15,5-43,0$ \\
\hline Independencia & 10 & 25,9 & $13,2-46,2$ \\
\hline Providencia & 17 & 23,2 & $14,0-36,4$ \\
\hline Cauquenes & 7 & 22,2 & $9,7-44,0$ \\
\hline Rancagua & 37 & 19,2 & $13,8-26,2$ \\
\hline Padre Las Casas & 12 & 19,0 & $10,3-32,4$ \\
\hline Las Condes & 35 & 19,0 & $13,4-26,1$ \\
\hline Macul & 14 & 18,8 & $10,7-30,8$ \\
\hline Peñalolén & 37 & 17,2 & $12,3-23,5$ \\
\hline Lo Barnechea & 14 & 16,4 & $9,3-26,8$ \\
\hline La Reina & 11 & 16,1 & $8,5-27,9$ \\
\hline
\end{tabular}

incidencia de EK incluyen diferencias étnicas que reflejen diversa susceptibilidad genética entre regiones de Chile, factores socio-económicos que determinen mayor acceso y oportunidad diagnóstica, así como también factores ambientales y meteorológicos. 
Los registros de egresos hospitalarios constituyen un buen marcador de incidencia de EK ya que la mayoría de los casos son diagnosticados en el período de evolución intrahospitalaria en el contexto de un síndrome febril o se hospitalizan para recibir tratamiento específico, por lo que han sido utilizados internacionalmente para estimar la incidencia de $\mathrm{EK}^{14,15}$. De acuerdo con lo publicado previamente por nuestro grupo, la incidencia de EK en el país ha aumentado durante los últimos años ${ }^{13}$, concentrándose la mayoría de los egresos hospitalarios por EK en la zona centro de Chile y particularmente en la RM. Los egresos por EK dentro de la RM se distribuyen, a su vez, de forma asimétrica, presentando el SSMO más de $40 \%$ de los egresos hospitalarios por EK en la región.

Estudios internacionales previos han reportado variaciones geográficas en la incidencia de EK, encontrándose las mayores tasas de EK en países de Asia ${ }^{3,7}$. Las diferentes incidencias de EK entre países, podrían ser explicadas, en parte, por una distinta composición étnica. La incidencia de EK es mayor entre los niños de etnia asiática en países con mayor variedad étnica como Estados Unidos de América (E.U.A.) ${ }^{14}$, lo que podría estar asociado a una susceptibilidad genética particu$\operatorname{lar}^{16,17}$. Nuestro país cuenta con una etnia relativamente homogénea de norte a sur, resultante de la mezcla entre europeos, principalmente españoles, y pueblos originarios americanos, por lo que la marcada diferencia encontrada en la incidencia de EK a lo largo de Chile no sería explicada por una variación étnica significativa entre las zonas norte, centro y sur del país. Sin embargo, destaca que la región de mayor incidencia del país sea La Araucanía y en particular que las dos comunas con mayor incidencia del país -muy por sobre la media nacional- tengan alta tasa de población mapuche. Las etnias indígenas chilenas como los mapuche están genéticamente relacionados con otros pueblos amerindios con ancestros comunes que migraron desde Asia ${ }^{18,19}$. La incidencia de EK en nativos de islas del Pacífico como Hawaii y Nueva Zelandia es mayor que en población caucásica y relativamente similar a la observada en población asiática ${ }^{20,21}$. Por otra parte, existe evidencia contradictoria respecto a esta asociación, ya que un estudio realizado en E.U.A. reportó tasas de incidencia de EK menores en niños de poblaciones nativas de Alaska y nativos americanos comparados con la población caucásica del país ${ }^{22}$. Estudios que utilicen como unidad de análisis casos individuales serían necesarios para confirmar la asociación entre las etnias amerindias chilenas y la EK.

En Chile no se han registrado brotes epidémicos de EK en los últimos años, salvo por un leve incremento de incidencia en el año 2005 de hasta 10,9 casos por 100.000 niños bajo 5 años ${ }^{13}$, muy por debajo de las altas incidencias reportadas durante brotes epidémicos en países como Japón y E.U.A. ${ }^{5,23}$. La distribución espacial de los casos de EK dentro de la RM, separados tanto por SS de residencia como por SS de egreso hospitalario, no se ha comportado siguiendo las características clásicas de una enfermedad infecciosa como ocurre con influenza y otros virus respiratorios, dado que para un área geográfica relativamente pequeña como la RM se esperaría una tasa de incidencia de EK con poca variación entre los distintos SS metropolitanos. El SSMO, que en el período estudiado concentró al 15,6 por ciento de la población bajo 18 años de edad en la RM, reportó más de un cuarto de los casos de EK en la región por SS de residencia y $45 \%$ de los casos de EK por SS de egreso hospitalario en la RM. El SSMO comprende al sector de mayor nivel socio-económico de la región y el país, presentando niveles de pobreza y población beneficiaria de FONASA significativamente menores que los otros SS de la región. Por otra parte, SS con una alta cantidad de casos entre sus habitantes como el suroriente, presentan un número significativamente menor de casos al medirlos según SS de egreso, posiblemente reflejo de una carencia de camas hospitalarias o acceso a ellas durante el período de estudio. Estudios norteamericanos han reportado previamente que en la EK se presentaría con mayor frecuencia en población de mayor nivel socio-económico, evaluado tanto por el ingreso promedio familiar como por el nivel educacional de los padres ${ }^{5,6,24,25}$. En cambio, en otros países como Inglaterra y Japón no se ha observado una asociación de nivel socio-económico con incidencia de $\mathrm{EK}^{12,15}$.

La concentración de los casos de EK en el SSMO podría indicar una mayor oportunidad diagnóstica en sectores de la población con mejores posibilidades de acceso a la salud privada de nuestro país ya que las diferencias en la distribución de los casos de EK en la RM se acentúan aún más cuando se considera el SS de egreso hospitalario. Esto sugiere que parte de los casos de EK que egresan del SSMO provienen de otras comunas de la región y buscan atención médica en zonas distintas a la de residencia en forma espontánea o estarían siendo derivados desde otros SS de la región y del país con menor acceso a sub-especialistas pediátricos o a métodos diagnósticos específicos como la ecocardiografía, para completar su proceso diagnóstico o bien para recibir tratamiento con inmunoglobulina endovenosa. Diferencias en la incidencia de EK entre la población suscrita al sistema de FONASA y la perteneciente al sistema de salud privado en Chile han sido previamente descritas ${ }^{13}$. Todo lo anterior apoya la idea de que en nuestro país, una desigualdad de oportunidad diagnóstica podría jugar un rol importante en las diferencias de incidencias encontradas entre las diversas regiones de Chile.

La RIE es una medida estadística que nos permite determinar diferencias significativas entre regiones del país y sospechar la existencia de sub-diagnóstico de una enfermedad por debajo de la tasa de incidencia nacional. 
De acuerdo con los datos registrados en las bases de datos de egresos hospitalarios correspondientes al período estudiado, en nuestro país sólo en la RM y La Araucanía se estaría diagnosticando EK con una tasa por sobre la tasa nacional, mientras que en las regiones de Tarapacá, Antofagasta, Atacama, Maule, Bío-Bío, Los Lagos y Magallanes habría menos casos que la media nacional o posiblemente un sub-diagnóstico de éstos. La EK es una enfermedad que requiere un alto índice de sospecha, lo que depende de la experiencia de los pediatras regionales con la enfermedad y de la presencia de sub-especialistas pediátricos. Con estos antecedentes, parece relevante reforzar la educación continua y reforzar la necesidad de especialistas en EK en las regiones del país que presentan tasas bajo la media nacional.

La vasta geografía de Chile determina importantes diferencias medio-ambientales y meteorológicas entre las diferentes regiones. La EK presenta una clara estacionalidad en muchos países del mundo incluyendo Japón, E.U.A. y Chile ${ }^{26}$. Estudios han reportado una asociación de cambios en vientos troposféricos con incidencia regional de EK en Japón y E.U.A., sospechándose de un putativo agente aerosolizado que es transportado por el viento desde una zona en el noreste de China ${ }^{27,28}$. El efecto de variables meteorológicas de escala mediana y grande como los vientos troposféricos y la oscilación del sur El Niño también presentan una asociación con incidencia de EK en la Región Metropolitana ${ }^{29}$, corroborando así los datos del Hemisferio Norte. Aunque esto no ha sido estudiado aún para otras regiones de Chile, es posible que las variaciones meteorológicas determinen variaciones en incidencia interna para un país con gran variación latitudinal y diferencias climáticas como Chile.

El tratamiento de la EK con inmunoglobulinas ha demostrado ser efectivo si es usado dentro de una ventana de tiempo limitada, que comprende desde el $5^{\circ}$ al $10^{\circ}$ día desde el inicio de los síntomas. Su uso puede reducir la aparición de aneurismas coronarios hasta 3 a $5 \%$ si es usado dentro de esta ventana de oportunidad terapéutica $^{2}$. Series chilenas de EK provenientes de distintos centros han mostrado que $35 \%$ de 575 pacientes con EK presentaron alteraciones coronarias, aunque se trata de series que abarcan distintos períodos de tiempo y no estandarizadas en cuanto a diagnóstico, realización de ecocardiografía y tratamiento con inmunoglobulina $a^{30-37}$. La ventana de oportunidad terapéutica de la EK en que el uso de inmunoglobulina es efectivo en prevenir el desarrollo de lesiones coronarias en pacientes con EK hace aún más importante el tener un bajo umbral de sospecha y disponer de las herramientas necesarias para evaluar de manera eficiente probables casos de EK. Los resultados de un registro multicéntrico latinoamericano de EK (REKAMLATINA) serán claves para obtener datos de las características clínicas y de respuesta al tratamiento de niños con EK en Chile ${ }^{38}$.

En conclusión, el presente estudio muestra la epidemiología de la EK a lo largo de Chile, reportando importantes diferencias de incidencia dentro de nuestro país. Estas podrían ser explicadas por una desigualdad en la oportunidad diagnóstica de EK, así como diferencias de nivel socio-económico, susceptibilidad genética y variables geográficas y meteorológicas del lugar de residencia de pacientes afectados.

\section{Resumen}

Introducción: La incidencia de enfermedad de Kawasaki (EK) en Chile ha aumentado; sin embargo, la distribución detallada de los casos en el país es desconocida. Objetivo: Describir la epidemiología de la EK en Chile entre 2001 y 2011 y estudiar la distribución geográfica de los casos de EK a lo largo del país. Métodos: Se obtuvieron los egresos hospitalarios por EK (ICD10 M30.3) en $<18$ años de bases de datos nacionales. Se calcularon las tasas de egreso hospitalario por EK en $<5$ años por 100.000 habs. para cada comuna, servicio de salud (SS) y región como estimación de incidencia de EK. Resultados: Se registraron 1.404 egresos por EK. La tasa de incidencia de EK fue de 8,7 con un aumento significativo desde el trienio 2001-2003 al trienio 20092011 desde 5,9 a 10,4 ( $<<0,001)$. Las regiones con mayores egresos por EK fueron: IX (Araucanía), Región Metropolitana y VI (O’Higgins) con tasas de 12,4; 11,1 y 10,5 , respectivamente. Las regiones con menores egresos por EK fueron: III (Atacama), II (Antofagasta) y XII (Magallanes) con tasas de 0,8;3,9 y 4, respectivamente. El SS Metropolitano Oriente registró la tasa más alta de EK del país $(19,8)$ y concentró $23,9 \%$ de los egresos por EK del país. Conclusión: La EK en Chile se distribuye heterogéneamente concentrándose principalmente en la zona central y en el SS Metropolitano Oriente. Las variaciones geográficas de EK en Chile podrían estar asociadas a diferencias reales de incidencia o diferencias en oportunidad diagnóstica, acceso a especialistas y a centros terciarios de atención de salud. 


\section{Referencias bibliográficas}

1.- Kawasaki T. Acute febrile mucocutaneous syndrome with lymphoid involvement with specific desquamation of the fingers and toes in children. Arerugi 1967; 16: 178-222.

2.- Newburger J W, Takahashi M, Gerber M A, Gewitz M H, Tani L Y, Burns J C, et al. Diagnosis, treatment, and long-term management of Kawasaki disease: a statement for health professionals from the Committee on Rheumatic Fever, Endocarditis and Kawasaki Disease, Council on Cardiovascular Disease in the Young, American Heart Association. Circulation 2004; 110: 2747-71.

3.- Yanagawa H, Nakamura Y. International comparison of the epidemiology of Kawasaki disease. Nippon Rinsho 2008; 66: 237-45.

4.- Burns J C, Cayan D R, Tong G, Bainto E V, Turner C L, Shike H, et al. Seasonality and temporal clustering of Kawasaki syndrome. Epidemiology 2005; 16: 220-5.

5.- Dean A G, Melish M E, Hicks R, Palumbo N E. An epidemic of Kawasaki syndrome in Hawaii. J Pediatr 1982; 100: 552-7.

6.- Bell D M, Brink E W, Nitzkin J L, Hall C B, Wulff H, Berkowitz I D, et al. Kawasaki syndrome: description of two outbreaks in the United States. N Engl J Med 1981; 304: 1568-75.

7.- Nakamura Y, Yashiro M, Uehara R, Sadakane A, Tsuboi S, Aoyama Y, et al. Epidemiologic features of Kawasaki disease in Japan: results of the 2009-2010 nationwide survey. J Epidemiol 2012; 22: 216-21.

8.- Kao A S, Getis A, Brodine S, Burns J C. Spatial and temporal clustering of Kawasaki syndrome cases. Pediatr Infect Dis J 2008; 27 : 981-5.

9.- Fischer T K, Holman R C, Yorita K L, Belay E D, Melbye M, Koch A. Kawasaki syndrome in Denmark. Pediatr Infect Dis J 2007; 26: 411-5.

10.- Kushner H I, Macnee R P, Burns J C. Kawasaki disease in India: increasing awareness or increased incidence? Perspect Biol Med 2009; 52: 17-29.

11.- Park Y W, Han J W, Hong Y M, Ma J S, Cha S H, Kwon T C, et al. Epidemiological features of Kawasaki disease in Korea, 2006-2008. Pediatr Int 2011; 53: 36-9.

12.- Nagao Y. Decreasing fertility rate correlates with the chronological increase and geographical variation in incidence of Kawasaki disease in Japan. PLoS One 2013; 8: e67934.

13.- Borzutzky A, Hoyos-Bachiloglu R, Cerda J, Talesnik E. Rising hospitalization rates of Kawasaki disease in Chile between 2001 and 2007. Rheumatol Int 2012; 32: 2491-5.
14.- Holman R C, Belay E D, Christensen K Y, Folkema A M, Steiner C A, Schonberger L B. Hospitalizations for Kawasaki syndrome among children in the United States, 1997-2007.

Pediatr Infect Dis J. 2010; 29: 483-8.

15.- Harnden A, Mayon-White R, Perera R, Yeates D, Goldacre M, Burgner D. Kawasaki disease in England: ethnicity, deprivation, and respiratory pathogens. Pediatr Infect Dis J 2009; 28: 21-4.

16.- Burns J C, Shimizu C, González E, Kulkarni H, Patel S, Shike H, et al. Genetic variations in the receptor-ligand pair CCR5 and CCL3L1 are important determinants of susceptibility to Kawasaki disease. J Infect Dis 2005; 192: 344-9.

17.- Burgner D, Dávila S, Breunis W B, Ng S B, Li Y, Bonnard C, et al. A genome-wide association study identifies novel and functionally related susceptibility loci for Kawasaki disease. PLoS Genet 2009; 5: e1000319.

18.- Achilli A, Perego U A, Bravi C M, Coble M D, Kong Q P, Woodward S R, et al. The phylogeny of the four pan-American MtDNA haplogroups: implications for evolutionary and disease studies. PLoS One 2008; 3: e1764.

19.- García F, Moraga M, Vera S, Henríquez H, Llop E, Aspillaga E, et al. mtDNA microevolution in Southern Chile's archipelagos. Am J Phys Anthropol 2006; 129 : 473-81.

20.- Holman R C, Christensen K Y, Belay E D, Steiner C A, Effler P V, Miyamura J, et al. Racial/ethnic differences in the incidence of Kawasaki syndrome among children in Hawaii. Hawaii Med J 2010; 69: 194-7.

21.- Heaton P, Wilson N, Nicholson R, Doran J, Parsons A, Aiken G. Kawasaki disease in New Zealand. J Paediatr Child Health 2006; 42: 184-90.

22.- Holman R C, Belay E D, Clarke M J, Kaufman S F, Schonberger L B. Kawasaki syndrome among American Indian and Alaska Native children, 1980 through 1995. Pediatr Infect Dis J 1999; 18: 451-5.

23.- Yanagawa H, Nakamura Y, Kawasaki T, Shigematsu I. Nationwide epidemic of Kawasaki disease in Japan during winter of 1985-86. Lancet 1986; 2: 1138-9.

24.- Bronstein D E, Dille A N, Austin J P, Williams C M, Palinkas L A, Burns J C. Relationship of climate, ethnicity and socioeconomic status to Kawasaki disease in San Diego County, 1994 through 1998. Pediatr Infect Dis J 2000; 19: 1087-91.

25.- Ichida F, Fatica N S, O’Loughlin J E, Klein A A, Snyder M S, Levin A R, et al. Epidemiologic aspects of Kawasaki disease in a Manhattan hospital. Pediatrics 1989; 84 : 235-41.

26.- Burns J C, Herzog L, Fabri O, Tremoulet A H, Rodo X, Uehara R, et al. Seasonality of Kawasaki disease: a global perspective. PLoS One 2013; 8: e74529.

27.- Rodo X, Ballester J, Cayan D, Melish M E, Nakamura Y, Uehara R, et al. Association of Kawasaki disease with tropospheric wind patterns. Sci Rep 2011; 1: 152.

28.- Rodo X, Curcoll R, Robinson M, Ballester J, Burns J C, Cayan D R, et al. Tropospheric winds from northeastern China carry the etiologic agent of Kawasaki disease from its source to Japan. Proc Natl Acad Sci USA 2014; 111: 7952-7.

29.- Jorquera H, Borzutzky A, Hoyos-Bachiloglu R, García A. Association of Kawasaki disease with tropospheric winds in Central Chile: is wind-borne desert dust a risk factor? Environ Int 2015; 78: 32-8.

30.- Barría R A K, Stegmaier H, Heusser F, Cespedes P, Talesnik E. Kawasaki disease. An experience of 15 years. Santiago of Chile. J Clin Rheumatol 2006; 12: S73.

31.- Schonhaut L A K, Álvarez P, Herrera P. Cardiovascular complications in Kawasaki disease. Rev Chil Pediatr 2000; 71: 311-5.

32.- Martínez A S G, Toledo M I, Molina N, Collipal J, Peldoza T. Enfermedad de Kawasaki Región de la Araucanía 2003-2007. Rev Chil Pediatr 2007; 78: 623-84.

33.- Álvarez D M J, Suau T. Caracterización de Enfermedad de Kawasaki durante el año 2007 en Hospital Exequiel González Cortés. Rev Chil Pediatr 2007; 78: 623-84.

34.- García C A C, Lueg E, González M, Basaez E. Enfermedad de Kawasaki. Clinica y compromiso cardiovascular. Rev Chil Pediatr 1999; 70: 519-70.

35.- Cabello M G G, Arancibia R. Enfermedad de Kawasaki: experiencia local. Rev Chil Pediatr 2006; 77.

36.- Zepeda G G M, Lillo A, Jofré L, Hinrichsen M, Jara L, Rojas A, Muñoz A, Monteverde M. Enfermedad de Kawasaki. Experiencia de un hospital universitario. Rev Chil Pediatr 2006; 77 (6): 621-688. Resúmenes del XLVI Congreso Chileno de Pediatría. 21 al 25 de noviembre de 2006, Chile.

37.- Henríquez I E G, Miranda P, Doggenweiler P, Hinrichsen M, Soler P, Soto J. Kawasaki disease: 17 years of experience. Rev Chil Cardiol 2002; 21: 109-16.

38.- González-Mata A, Ulloa-Gutiérrez R, Brea J, Soza G, Tremoulet A H. Origin and importance of the Latin American Kawasaki Disease Network (REKAMLATINA) Rev Chilena Infectol 2014; 31: 330-2 